\title{
Some New Transformation Properties of the Nielsen Generalized Polylogarithm
}

\author{
Nina Shang, Qinghua Feng, and Huizeng Qin \\ Institute of Applied Mathematics, Shandong University of Technology, Zibo, Shandong 255049, China \\ Correspondence should be addressed to Huizeng Qin; qinhz_000@163.com
}

Received 20 March 2014; Accepted 13 May 2014; Published 12 June 2014

Academic Editor: Biren N. Mandal

Copyright ( 2014 Nina Shang et al. This is an open access article distributed under the Creative Commons Attribution License, which permits unrestricted use, distribution, and reproduction in any medium, provided the original work is properly cited.

Many of the properties of Nielsen generalized polylogarithm $S_{n, p}(z)$, for example, the special value and the transformation formulas, play important roles in the computation of higher order radiative corrections in quantum electrodynamics. In this paper, some transformation formulas of $z \rightarrow p(z), p(z)=1-z, 1 / z, 1 /(1-z), z /(z-1)$, and $(1-z) / z$ are obtained. In particular, the last three transformation formulas are new results so far in the literature. By use of these transformation formulas presented, new fast algorithms for Nielsen generalized polylogarithm $S_{n, p}(z)$ can be designed. For $s_{n, p}=S_{n, p}(1)$, a new recurrence formula is also given. The identities and the calculation of $\sigma_{n, p}$ and $a_{n, p}$ are also investigated.

\section{Introduction}

The Nielsen generalized polylogarithm, introduced by the Danish mathematician Niels Nielsen, reads as follows:

$$
S_{n, p}(z)=\frac{(-1)^{n+p-1}}{(n-1) ! p !} \int_{0}^{1} \frac{\ln ^{n-1} t \ln ^{p}(1-z t)}{t} d t
$$

in which $z$ is a complex and $n$ and $p$ are positive integers. It is a generalization of the polylogarithm function $L i_{n}(z)$. In fact, $L i_{n+1}(z)=S_{n, 1}(z)$. In particular, the values of $S_{n, p}(x)$ for $x=$ $1, x=-1$, and $x=1 / 2$ are usually used in the theory of the generalized polylogarithms. According to Nielsen's notation as follows,

$$
\begin{gathered}
s_{n, p}=S_{n, p}(1), \quad \sigma_{n, p}=(-1)^{p} S_{n, p}(-1), \\
a_{n, p}=S_{n, p}\left(\frac{1}{2}\right),
\end{gathered}
$$

the following relationship exists:

$$
\begin{gathered}
s_{n, p}=s_{p, n}, \quad s_{n}=s_{n-1,1}=\zeta(n), \\
\sigma_{n}=\sigma_{n-1,1}=\eta(n)=\left(1-2^{1-n}\right) \zeta(n),
\end{gathered}
$$

where $\zeta(n)$ is Riemann zeta function and $\eta(n)$ is the Dirichlet eta function.
The Nielsen generalized polylogarithm plays an important role in quantum electrodynamics (see $[1,2])$. The properties of it can also find applications in group theory and geometry [3]. For the Nielsen generalized polylogarithm $S_{n, p}(z)$, Kölbig has done much in-depth and systematic study in $[4,5]$. For example, the transformations $z \rightarrow 1-z$ and $z \rightarrow 1 / z$ are given as follows:

$$
\begin{aligned}
S_{n, p}(z) & +\sum_{j=1}^{n} \frac{\ln ^{n-j} z}{(n-j) !} \\
& \times \sum_{k=1}^{p} \frac{(-1)^{p-k} \ln ^{p-k}(1-z)}{(p-k) !} S_{k, j}(1-z) \\
= & \sum_{j=1}^{n} \frac{\ln ^{n-j} z}{(n-j) !} s_{j, p}+\frac{(-1)^{p} \ln ^{p}(1-z) \ln ^{n} z}{p ! n !}, \\
S_{n, p}(z) & (-1)^{n} \sum_{m=0}^{p-1} \frac{\ln m(-z)}{m !} \sum_{k=m}^{p-1}(-1)^{k} C_{n+k-m-1}^{k-m} S_{n+k-m, p-k}\left(\frac{1}{z}\right) \\
& +(-1)^{p} \sum_{m=0}^{n-1} \frac{\ln ^{m}(-z)}{m !} C_{n-m, p} \\
& +(-1)^{p} \ln _{n+p}(-z),
\end{aligned}
$$


where

$$
\begin{aligned}
C_{m, p}= & \sum_{k=1}^{p-1}(-1)^{m+p-k-1} C_{m+k-1}^{k} \\
& \times \sum_{j=0}^{[(m+k-1) / 2]} \frac{(-1)^{j} \pi^{2 j}}{(2 j) !} s_{m+k-2 j, p-k} \\
& +(-1)^{m+p-1}\left(1-(-1)^{m}\right) \sum_{j=0}^{[(m-1) / 2]} \frac{(-1)^{j} \pi^{2 j}}{(2 j) !} s_{m-2 j, p} \\
& +(-1)^{[(m+p) / 2]+n} \frac{\varepsilon_{m+p} \pi^{m+p}}{(m+p)(m-1) ! p !}
\end{aligned}
$$

(see (5.3) and (5.12) in [5]). In [5], the author also noted that by repeated use of (4)-(6) it is possible to find formulae for

$$
S_{n, p}\left(\frac{1}{1-z}\right), \quad S_{n, p}\left(\frac{z-1}{z}\right), \quad S_{n, p}\left(\frac{z}{z-1}\right),
$$

which only contains generalized polylogarithms apart from logarithms and known constants. These formulae soon become very complicated and a more systematic investigation needs to be done later.

In this paper, we use different methods from that in [5] to give the properties of the transformations $z \rightarrow p(z), p(z)=$ $1-z, 1 /(1-z), z /(z-1)$, and $(1-z) / z$ for the Nielsen generalized polylogarithm $S_{n, p}(z)$. For $s_{n, p}=S_{n, p}(1)$, we also give a new recurrence formula. Finally, we consider the identities and the calculation of $\sigma_{n, p}, a_{n, p}$ and high accuracy and fast calculation of $S_{n, p}(z)$.

\section{The Properties of the Transformations $z \rightarrow$ $1-z, 1 /(1-z), z /(z-1)$, and $(1-z) / z$}

For the Nielsen generalized polylogarithm, in [5], by use of the following results (see [6, (2.15), (2.16)]),

$$
\begin{gathered}
S_{n, p}(z)=\sum_{j=0}^{n-1} \frac{\ln ^{j} z}{j !} L_{n-j, p}(z), \\
L_{n, p}(z)=\sum_{j=0}^{n-1} \frac{(-1)^{j} \ln ^{j} z}{j !} S_{n-j, p}(z),
\end{gathered}
$$

where

$$
L_{n, p}(z)=\frac{(-1)^{n+p-1}}{(n-1) ! p !} \int_{0}^{z} \frac{\ln ^{n-1} t \ln ^{p}(1-t)}{t} d t
$$

and some identities, the transformation properties (4)-(6) were obtained.

In this section, we use different methods to give the properties of the transformations $z \rightarrow 1-z, 1 / z, 1 /(1-$ $z), z /(z-1)$, and $(1-z) / z$. First we introduce the following function:

$$
\begin{aligned}
S_{n, p}(z ; x, y)= & \frac{(-1)^{n+p-1}}{(n-1) ! p !} \\
& \times \int_{0}^{1} t^{x-1}(1-z t)^{y-1} \ln ^{n-1} t \ln ^{p}(1-z t) d t, \\
s_{n, p}(x, y)= & S_{n, p}(1, x, y) .
\end{aligned}
$$

It is easy to get the following relation:

$$
\begin{gathered}
S_{n, p}(z)=S_{n, p}(z ; 0,1), \\
s_{n, p}=s_{n, p}(0,1), \\
B(z ; x, y)=z^{x} S_{1,0}(z ; x, y), \\
B(x, y)=S_{1,0}(1 ; x, y), \\
\frac{\partial^{n+p-1}}{\partial x^{n-1} \partial y^{p}} B(x, y)=(-1)^{n+p-1}(n-1) ! p ! s_{n, p}(x, y), \\
S_{n, p}(z ; x, y)=\frac{(-1)^{n+p-1}}{(n-1) ! p !} \frac{\partial^{n+p-1}}{\partial x^{n-1} \partial y^{p}} S_{1,0}(z ; x, y) \\
=\frac{(-1)^{n+p-1}}{(n-1) ! p !} \frac{\partial^{n+p-1}}{\partial x^{n-1} \partial y^{p}}\left[z^{-x} B(z ; x, y)\right], \\
\frac{\partial^{n+p-1}}{\partial x^{n-1} \partial y^{p}} B(z ; x, y)=(-1)^{n+p-1}(n-1) ! p ! z^{x} \\
\times \sum_{j=1}^{n} \frac{(-1)^{n-j} \ln ^{n-j} z}{(n-j) !} S_{j, p}(z ; x, y),
\end{gathered}
$$

where $B(x, y)$ and $B(z ; x, y)$ are the Beta function and the incomplete Beta function, respectively.

Lemma 1. When $x, y>0$, for $S_{1,0}(z, x, y)$, there are the following properties:

(1) $z \neq r(r<0$ or $r>1)$ :

$$
\begin{aligned}
& S_{1,0}(z, x, y) \\
& =z^{-x} S_{1,0}(1 ; x, y)-z^{-x}(1-z)^{y} S_{1,0}(1-z, y, x) . \\
& \quad(2) z \neq r(r>0): \\
& S_{1,0}(z, x, y) \\
& =z^{-x} S_{1,0}(1 ; x, y)-(-1)^{H(z) y} z^{-x} S_{1,0}(1 ; 1-x-y, y) \\
& \quad-(-z)^{y-1} S_{1,0}\left(\frac{1}{z} ; 1-x-y, y\right),
\end{aligned}
$$

where

$$
H(z)= \begin{cases}-1, & \operatorname{Im} z>0 \text { or } z<0 \\ 1, & \operatorname{Im} z<0 \text { or } z>1\end{cases}
$$


(3) $z \neq r(r \geq 1$ or $r<0)$ :

$$
\begin{aligned}
S_{1,0}(z, x, y)= & z^{-x} S_{1,0}(1 ; x, y)-z^{-x}\left(\frac{1-z}{z}\right)^{y} \\
& \times S_{1,0}\left(\frac{z-1}{z} ; y, 1-x-y\right):
\end{aligned}
$$

(4) $z \neq r(r \geq 1)$ :

$$
S_{1,0}(z, x, y)=(1-z)^{-x} S_{1,0}\left(\frac{z}{z-1} ; x, 1-x-y\right)
$$

(5) $z \neq r(r>0)$ :

$$
S_{1,0}(z, x, y)
$$

$$
\begin{aligned}
= & (-z)^{-x} S_{1,0}(1 ; 1-x-y, x)-\left(\frac{z-1}{z}\right)^{x}(1-z)^{y-1} \\
& \times S_{1,0}\left(\frac{1}{1-z} ; 1-x-y, x\right) .
\end{aligned}
$$

Proof. (1) By (10) we have

$$
\begin{aligned}
S_{1,0}(z ; x, y) & =\int_{0}^{1} t^{x-1}(1-z t)^{y-1} d t \\
& =z^{-x} \int_{0}^{z} t^{x-1}(1-t)^{y-1} d t \\
& =z^{-x}\left(s_{1,0}(x, y)+\int_{1}^{z} t^{x-1}(1-t)^{y-1} d t\right) \\
& =z^{-x}\left(s_{1,0}(x, y)-\int_{0}^{1-z} t^{y-1}(1-t)^{x-1} d t\right) \\
& =z^{-x}\left(s_{1,0}(x, y)-B(1-z, y, x)\right) .
\end{aligned}
$$

So (12) holds.

(2) For $B(z ; x, y)$ we have

$$
B(z ; x, y)=\left\{\begin{array}{r}
B(i ; x, y)+\int_{i}^{z} t^{x-1}(1-t)^{y-1} d t, \\
B(-i ; x, y)+\int_{-i}^{z} t^{x-1}(1-t)^{y-1} d t, \\
\operatorname{Im} z<0 \text { or } z>1,
\end{array}\right.
$$

where the second integral path does not go through the real axes. By using the variable substitution, we have

$$
\begin{array}{r}
\int_{i}^{z} t^{x-1}(1-t)^{y-1} d t=(-1)^{-y} \int_{-i}^{1 / z} t^{-x-y}(1-t)^{y-1} d t, \\
\operatorname{Im} z>0, \\
\int_{-i}^{z} t^{x-1}(1-t)^{y-1} d t=(-1)^{y} \int_{i}^{1 / z} t^{-x-y}(1-t)^{y-1} d t,
\end{array}
$$

Substituting (20) into (19) we get that

$$
\begin{aligned}
& B(z ; x, y) \\
& =\left\{\begin{array}{c}
B(i, x, y)-(-1)^{-y} B(-i, 1-x-y, y) \\
+(-1)^{-y} B\left(\frac{1}{z} ; 1-x-y, y\right), \\
\operatorname{Im} z>0 \\
B(-i, x, y)-(-1)^{y} B(i, 1-x-y, y) \\
+(-1)^{y} B\left(\frac{1}{z} ; 1-x-y, y\right), \\
\operatorname{Im} z<0 \text { or } z>1 .
\end{array}\right.
\end{aligned}
$$

Letting $z \rightarrow 1$, we obtain that

$$
\begin{aligned}
& B(x, y)-(-1)^{-y} B(1-x-y, y) \\
&= B(i, x, y)-(-1)^{-y} B(-i, 1-x-y, y), \\
& B(x, y)-(-1)^{y} B(1-x-y, y) \\
&=B(-i, x, y)-(-1)^{y} B(i, 1-x-y, y) .
\end{aligned}
$$

By (22) and (21) we get that

$$
\begin{aligned}
B(z ; x, y)= & B(x, y)+(-1)^{H(z) y} \\
& \times\left(B(1-x-y, y)-B\left(\frac{1}{z} ; 1-x-y, y\right)\right) .
\end{aligned}
$$

Then by (11) one can see that (13) holds. By (13) and (12) we deduce that (15) holds.

Fully in accordance with the discussion of (2), we can also get that (15)-(17) hold.

By means of the application of Hadamard integral (the Hadamard finite part integral or neutrix calculus; see [6-8]) and integration by parts we have

$$
\begin{array}{r}
\frac{\partial^{n-1}}{\partial x^{n-1}} B(z, 0,1)=\int_{0}^{z} t^{-1} \ln ^{n-1} t d t=\frac{\ln ^{n} z}{n}, \\
\frac{\partial^{n-1}}{\partial x^{n-1}} B(0,1)=0,
\end{array}
$$

$$
S_{n, 0}(z)=\left.\frac{(-1)^{n-1}}{(n-1) !} \frac{\partial^{n-1}}{\partial x^{n-1}}\left[z^{-x} B(z ; x, y)\right]\right|_{x=0, y=1}=\frac{\ln ^{n} z}{n !},
$$

$$
s_{n, 0}=0,
$$




$$
\begin{gathered}
z S_{n+1, p-1}(z, 1,0)=S_{n, p}(z), \\
z S_{1, p-1}(z, 1,0)=\frac{(-1)^{p} \ln ^{p}(1-z)}{p !}, \\
S_{n, p}(z, 0,0)=S_{n, p}(z)+S_{n-1, p+1}(z), \\
s_{n, p}(0,0)=s_{n, p}+s_{n-1, p+1}, \quad n>1, p>0, \\
S_{1, p}(z, 0,0)=S_{1, p}(z)+\frac{(-1)^{p+1} \ln ^{p+1}(1-z)}{(p+1) !}, \\
s_{1, p}(0,0)=s_{1, p}, \quad p>0 \\
S_{1,0}(z, 0,0)=-\ln (1-z), \quad s_{1,0}(0,0)=0, \\
S_{n, 0}(z, 0,0)=S_{n-1,1}(z), \quad s_{n, 0}(0,0)=s_{n-1,1}, \\
n>1 .
\end{gathered}
$$

By Lemma 1, (24), and (25), we will consider the transformation properties of $S_{n, p}(z)$ on $z$. First we prove (4) in another approach.

Theorem 2. When the integers $n, p \geq 1$, (4) for the transformation $z \rightarrow 1-z$ holds for all complexes $z$.

Proof. By (11) and (12), we have

$$
\begin{aligned}
S_{n, p}(z, x, y)= & z^{-x} \sum_{j=1}^{n} \frac{\ln ^{n-j} z}{(n-j) !} s_{j, p}(x, y) \\
& -z^{-x}(1-z)^{y} \sum_{j=1}^{n} \frac{\ln ^{n-j} z}{(n-j) !} \\
& \times \sum_{k=0}^{p} \frac{(-1)^{p-k} \ln ^{p-k}(1-z)}{(p-k) !} \\
& \times S_{k+1, j-1}(1-z, y, x) .
\end{aligned}
$$

In (26), letting $y=1, x=0$ and using (25), we have

$$
\begin{aligned}
S_{n, p}(z)= & \sum_{j=1}^{n} \frac{\ln ^{n-j} z}{(n-j) !} s_{j, p}-(-1)^{p} \\
& \times \sum_{j=1}^{n} \frac{(-1)^{j}}{(n-j) ! j !} \frac{\ln ^{n} z \ln ^{p}(1-z)}{p !} \\
& -\sum_{j=1}^{n} \frac{\ln ^{n-j} z}{(n-j) !} \sum_{k=1}^{p} \frac{(-1)^{p-k} \ln ^{p-k}(1-z)}{(p-k) !} \\
& \times S_{k, j}(1-z),
\end{aligned}
$$

where $z$ can be extended to the whole complex plane. Then by (27) and $\sum_{j=1}^{n}\left((-1)^{j} /(n-j) ! j !\right)=-1 / n$ !, we deduce that (4) holds.
Theorem 3. When the integers $n, p \geq 1$, for the transformation $z \rightarrow 1 / z$, there is

$$
\begin{aligned}
S_{n, p}(z)= & \sum_{j=1}^{n} \frac{\ln ^{n-j} z}{(n-j) !} s_{j, p} \\
& +\frac{1}{(n-1) !} \sum_{k=0}^{p} \frac{(-1)^{p-k} \ln ^{p-k}(-z) \ln ^{n+k} z}{k !(p-k) !(n+k)} \\
& +(-1)^{n} \sum_{k=1}^{p} \frac{(-1)^{p-k} \ln ^{p-k}(-z)}{(p-k) !} \\
& \times \sum_{v=0}^{k-1}(-1)^{v} C_{n+v-1}^{v} S_{n+v, k-v}\left(\frac{1}{z}\right) \\
& -\sum_{j=1}^{n} \frac{(-1)^{j} \ln ^{n-j} z}{(n-j) !} \sum_{k=1}^{p} \frac{(-\pi i H(z))^{p-k}}{(p-k) !} \\
& \times \sum_{v=0}^{k-1}(-1)^{v} C_{j+v-1}^{v} s_{j+v, k-v},
\end{aligned}
$$

where $z \neq 1, i=\sqrt{-1}$.

Proof. By (11) and (13) and noting $\ln (-1)=\pi i$, we have

$$
\begin{aligned}
S_{n, p}(z, x, y)= & \frac{1}{z^{x}} \sum_{j=1}^{n} \frac{\ln ^{n-j} z}{(n-j) !} s_{j, p}(x, y) \\
& +\frac{(-1)^{n+p}}{(-z)^{1-y}} \sum_{k=0}^{p} \frac{\ln ^{p-k}(-z)}{(p-k) !} \\
& \times C_{n+k-1}^{k} S_{n+k, 0}\left(\frac{1}{z} ; 1-x-y, y\right) \\
& +\frac{(-1)^{n}}{(-z)^{1-y}} \sum_{k=1}^{p} \frac{(-1)^{p-k} \ln { }^{p-k}(-z)}{(p-k) !} \\
& \times \sum_{v=0}^{k-1}(-1)^{v} C_{n+v-1}^{v} S_{n+v, k-v}\left(\frac{1}{z} ; 1-x-y, y\right) \\
& +\frac{(-1)^{H(z) y}}{z^{x}} \sum_{j=1}^{n} \frac{(-1)^{j} \ln ^{n-j} z}{(n-j) !} \sum_{k=0}^{p} \frac{(-\pi i H(z))^{p-k}}{(p-k) !} \\
& \times \sum_{v=0}^{k}(-1)^{v} C_{j+v-1}^{v} s_{j+v, k-v}(1-x-y, y) .
\end{aligned}
$$

In (29), letting $x=0, y=1$ and using (24), we get that (28) holds. 
Theorem 4. When the integers $n, p \geq 1$, for the transformation $z \rightarrow(z-1) / z$, there is

$$
\begin{aligned}
S_{n, p}(z) & +(-1)^{n} \sum_{j=1}^{n} \frac{(-\ln z)^{n-j}}{(n-j) !} \sum_{k=1}^{p} \frac{(-\ln (1-z) / z)^{p-k}}{(p-k) !} \\
& \times \sum_{v=0}^{k-1}(-1)^{v} C_{j+v-1}^{v} S_{k-v, j+v}\left(\frac{z-1}{z}\right) \\
= & \sum_{j=1}^{n} \frac{\ln ^{n-j} z}{(n-j) !} s_{j, p}+(-1)^{n} \\
& \times \sum_{k=0}^{p} \frac{(-\ln (1-z) / z)^{p-k}(-\ln z)^{n+k}}{(p-k) !(k+n) !}
\end{aligned}
$$

where $z \neq r(r<0)$ and $z \neq 1$.

Proof. By (11) and (15) we have

$$
\begin{aligned}
S_{n, p}(z, x, y) \\
=z^{-x} \sum_{j=1}^{n} \frac{\ln ^{n-j} z}{(n-j) !} s_{j, p}(x, y) \\
\quad-\frac{((1-z) / z)^{y}}{z^{x}} \sum_{j=1}^{n} \frac{(-\ln z)^{n-j}}{(n-j) !} \sum_{k=0}^{p} \frac{(-\ln (1-z) / z)^{p-k}}{(p-k) !} \\
\quad \times \sum_{v=0}^{k}(-1)^{v} C_{j+v-1}^{v} S_{k+1-v, j+v-1}\left(\frac{z-1}{z} ; y, 1-x-y\right) .
\end{aligned}
$$

In (31), letting $y=1, x=0$ and by (25), we have

$$
\begin{aligned}
S_{n, p}( & z) \\
= & \sum_{j=1}^{n} \frac{\ln ^{n-j} z}{(n-j) !} s_{j, p}-(-1)^{n} \\
& \times \sum_{k=0}^{p} \frac{(-1)^{p-k} \ln ^{p-k}((1-z) / z)(-1)^{n+k} \ln ^{n+k} z}{(p-k) ! k !} \\
& \times \sum_{j=1}^{n} \frac{(-1)^{j}}{(n-j) !(j-1) !(j+k)} \\
& -(-1)^{n} \sum_{j=1}^{n} \frac{(-1)^{n-j} \ln { }^{n-j} z}{(n-j) !} \\
& \times \sum_{k=1}^{p} \frac{(-1)^{p-k} \ln ^{p-k}(1-z) / z}{(p-k) !} \\
& \times \sum_{v=0}^{k-1}(-1)^{v} C_{j+v-1}^{v} S_{k-v, j+v}\left(\frac{z-1}{z}\right) .
\end{aligned}
$$

Noting $\sum_{j=1}^{n}\left((-1)^{j} /(n-j) !(j-1) !(j+k)\right)=-k ! /(k+n) !$, one can see that (30) holds.
Theorem 5. When the integers $n, p \geq 1$, for the transformation $z \rightarrow z /(z-1)$ and $z \rightarrow 1 /(1-z)$, there is

$$
\begin{aligned}
S_{n, p}(z) & -(-1)^{p} \\
& \times \sum_{j=1}^{n} \frac{\ln ^{n-j}(1-z)}{(n-j) !} \sum_{u=0}^{j-1} C_{p+u-1}^{u}(-1)^{u} S_{j-u, p+u}\left(\frac{z}{z-1}\right) \\
= & \frac{(-1)^{p} \ln ^{n+p}(1-z)}{(n+p) !}
\end{aligned}
$$

where $z \neq r(r \geq 1)$, and

$$
\begin{aligned}
S_{n, p}( & (z) \\
& +(-1)^{p} \sum_{k=1}^{p} \frac{\ln ^{p-k}(1-z)}{(p-k) !} \\
& \times \sum_{j=1}^{n} \frac{(-1)^{n-j} \ln ^{n-j}(z-1) / z}{(n-j) !} \\
& \times \sum_{u=0}^{j-1} C_{k+u-1}^{u}(-1)^{u} S_{k+u, j-u}\left(\frac{1}{1-z}\right) \\
= & (-1)^{p} \sum_{j=1}^{n} \frac{\ln ^{n-j}(-z)}{(n-j) !} \\
& \times \sum_{u=0}^{j-1} C_{p+u}^{u}(-1)^{u} s_{p+u, j-u}+(-1)^{p} \\
& \times \sum_{j=0}^{n} \frac{(-1)^{n-j} \ln ^{n-j}((z-1) / z) \ln { }^{p+j}(1-z)}{(n-j) !(p+j) !}
\end{aligned}
$$

where $z \neq r(0 \leqslant r \leqslant 1)$.

Proof. By (11), (16), and (17) we obtain that

$$
\begin{aligned}
& S_{n, p}(z, x, y) \\
&=-\frac{(-1)^{p}}{(1-z)^{x}} \sum_{j=1}^{n} \frac{(-1)^{j} \ln ^{n-j}(1-z)}{(n-j) !} \\
& \times \sum_{u=0}^{j-1} C_{p+j-u}^{j-u}(-1)^{u} S_{1+u, p+j-1-u}\left(\frac{z}{z-1} ; x, 1-x-y\right), \\
& S_{n, p}(z, x, y) \frac{(-1)^{p}}{(-z)^{x}} \sum_{j=1}^{n} \frac{\ln { }^{n-j}(-z)}{(n-j) !} \\
& \quad \times \sum_{u=0}^{j-1} C_{p+u}^{u}(-1)^{u} s_{p+u+1, j-1-u}(1-x-y, x) \\
& \quad-\frac{(-1)^{p}((z-1) / z)^{x}}{(1-z)^{1-y}} \sum_{k=0}^{p} \frac{\ln ^{p-k}(1-z)}{(p-k) !}
\end{aligned}
$$




$$
\begin{aligned}
& \times \sum_{j=1}^{n} \frac{(-\ln (z-1) / z)^{n-j}}{(n-j) !} \\
& \times \sum_{u=0}^{j-1} C_{k+u}^{u}(-1)^{u} S_{k+u+1, j-1-u}\left(\frac{1}{1-z} ; 1-x-y, x\right) .
\end{aligned}
$$

In (35), letting $y=1, x=0$ and using (25), we can deduce that (33) and (34) hold.

\section{The Recursive Formula on $s_{n, p}=S_{n, p}(1)$}

For $s_{n, p}=S_{n, p}(1)$ in Theorems 2-5, we can give the following recursive formula.

Theorem 6. For the positive integers $n, p \geq 1$, there is

$$
\begin{aligned}
s_{n, p}= & \frac{C_{n+p}^{n} \zeta(n+p)}{n+p} \\
& -\frac{1}{p} \sum_{i=1}^{n-1} \sum_{j=1}^{p-1} C_{n+p-i-j-1}^{n-i} s_{i, j} \zeta(n+p-i-j) .
\end{aligned}
$$

Proof. By calculating $p$-order partial derivatives on $b$ for $B(a, b)$, we have

$$
\begin{aligned}
\frac{\partial^{p}}{\partial b^{p}} B(a, b) & \\
= & \frac{\partial^{p-1}}{\partial b^{p-1}}(B(a, b)(\psi(b)-\psi(a+b))) \\
= & \sum_{j=0}^{p-1} C_{p-1}^{j} \frac{\partial^{j}}{\partial b^{j}} B(a, b)\left(\psi^{(p-1-j)}(b)-\psi^{(p-1-j)}(a+b)\right) \\
\frac{\partial^{p}}{\partial b^{p}} & \left.B(a, b)\right|_{b=1} \\
= & \left.\sum_{j=0}^{p-1} C_{p^{j-1}} \frac{\partial^{j}}{\partial b^{j}} B(a, b)\right|_{b=1} \\
& \times\left(\psi^{(p-1-j)}(1)-\psi^{(p-1-j)}(a+1)\right)
\end{aligned}
$$

where $\psi(x)$ is the digamma function defined as

$$
\psi(x)=\frac{d}{d x} \ln \Gamma(x)=-\gamma-\frac{1}{x}+\sum_{m=1}^{\infty}\left(\frac{1}{m}-\frac{1}{m+x}\right) .
$$

By calculating $n-1$-order partial derivatives on $a$ in (38), we have

$$
\begin{aligned}
& \left.\frac{\partial^{n+p-1}}{\partial a^{n-1} \partial b^{p}} B(a, b)\right|_{b=1} \\
& =\sum_{j=1}^{p-1} C_{p-1}^{j}\left(\psi^{(p-1-j)}(1)-\psi^{(p-1-j)}(a+1)\right) \\
& \quad \times\left.\frac{\partial^{n+j-1}}{\partial a^{n-1} \partial b^{j}} B(a, b)\right|_{b=1} \\
& \quad+\frac{\partial^{n-1}}{\partial a^{n-1}}\left[\left.\left(\psi^{(p-1)}(1)-\psi^{(p-1)}(a+1)\right) B(a, b)\right|_{b=1}\right] \\
& \quad-\sum_{i=0}^{n-2 p-1} \sum_{j=1}^{i} C_{n-1}^{i} C_{p-1}^{j} \psi^{(n+p-2-i-j)}(a+1) \\
& \quad \times\left.\frac{\partial^{i+j}}{\partial a^{i} \partial b^{j}} B(a, b)\right|_{b=1} .
\end{aligned}
$$

Using $\psi^{(k)}(1)=k !(-1)^{k+1} \zeta(k+1)$, we have

$$
\begin{gathered}
\left.\left(\psi^{(n+p-2-i-j)}(a+1) \frac{\partial^{i+j}}{\partial a^{i} \partial b^{j}} B(a, b)\right)\right|_{a=0, b=1} \\
=(n+p-2-i-j) !(-1)^{n+p-1} i ! j ! s_{i+1, j} \\
\times \zeta(n+p-1-i-j), \\
i \geq 0, \quad j \geq 1 .
\end{gathered}
$$

By the property of the Beta function, it holds that

$$
\begin{aligned}
& \left.\frac{\partial^{n-1}}{\partial a^{n-1}}\left(B(a, b)\left(\psi^{(p-1)}(1)-\psi^{(p-1)}(a+1)\right)\right)\right|_{a=0, b=1} \\
& \quad=\left.\frac{\partial^{n-1}}{\partial a^{n-1}}\left(\frac{\psi^{(p-1)}(1)-\psi^{(p-1)}(a+1)}{a}\right)\right|_{a=0} \\
& =-\frac{1}{n} \psi^{(n+p-1)}(1) \\
& =\frac{(-1)^{n+p-1}(n+p-1) !}{n} \zeta(n+p) .
\end{aligned}
$$

Combining (10), (11), and (38)-(42), we obtain that (36) holds.

Remark 7. In [5], there is the following result:

$$
s_{n, p}=(-1)^{n+p-1} \sum_{i=1}^{n-1} b_{n-i-1} \sum_{j=1}^{p-1} C_{i+j+1}^{j} b_{p-j} a_{i+j+1},
$$


where $a_{0}=b_{0}=1$, and

$$
\begin{gathered}
a_{k}=\frac{1}{k} \sum_{m=1}^{k}(-1)^{m+1} \zeta(m) a_{k-m}, \\
b_{k}=-\frac{1}{k} \sum_{m=1}^{k}(-1)^{m+1} \zeta(m) b_{k-m}, \quad k>0,
\end{gathered}
$$

where $\zeta(1)=\gamma, \gamma$ is Euler's constant. From the perspective of recurrence relation, one can see that (36) is simpler and clearer than (43).

\section{On the Identities of $\sigma_{n, p}$ and $a_{n, p}$ and Their Calculations}

In (28), letting $z=-1$ and using $\ln (-1)=\pi i$, we have

$$
\begin{gathered}
2 \sigma_{2 n-1, p}+\sum_{v=1}^{p-1} C_{2 n+v-2}^{v} \sigma_{2 n+v-1, p-v}=(-1)^{p} c_{2 n-1, p}, \\
\sum_{v=1}^{p-1} C_{2 n+v-1}^{v} \sigma_{2 n+v, p-v}=(-1)^{p-1} c_{2 n, p}
\end{gathered}
$$

where

$$
\begin{aligned}
c_{n, p}= & \sum_{j=1}^{n} \frac{(\pi i)^{n-j}}{(n-j) !} s_{j, p}+\frac{(\pi i)^{n+p}}{(n-1) ! p !(n+p)} \\
& -\sum_{j=1}^{n} \frac{(-1)^{j}}{(n-j) !} \sum_{k=1}^{p} \frac{(\pi i)^{n+p-j-k}}{(p-k) !} \sum_{v=0}^{k-1}(-1)^{v} C_{j+v-1}^{v} s_{j+v, k-v} .
\end{aligned}
$$

Using (3) and (36), from (45) and (46), we have

$$
\begin{aligned}
\sigma_{2 n+1,2}= & \left(\frac{2 n+1}{2^{2 n+3}}-n\right) \zeta(2 n+3) \\
& +\frac{1}{2} \sum_{j=1}^{n} \frac{(2 n+1-2 j)(-1)^{n-j} \pi^{2 n+2-2 j}}{(2 n+2-2 j) !} \zeta(2 j+1) \\
& -\sum_{j=1}^{n} \frac{(-1)^{n-j} \pi^{2 n-2 j}}{(2 n-2 j) !} \sum_{l=1}^{j} \zeta(2 l+1) \zeta(2 j+2-2 l), \\
& \sum_{j=1}^{n} \frac{(-1)^{j-1} \zeta(2 j)}{(2 n+1-2 j) ! \pi^{2 j}}=\frac{n}{(2 n+1) !}
\end{aligned}
$$

Here (48) is a new formula.
In (34) and (4) letting $z=2$ we have

$$
\begin{aligned}
S_{n, p}(2) & +(-1)^{p} \\
& \times \sum_{k=1}^{p} \frac{\ln ^{p-k}(-1)}{(p-k) !} \sum_{j=1}^{n} \frac{(-1)^{j} \ln ^{n-j} 2}{(n-j) !} \sum_{u=0}^{j-1} C_{k+u-1}^{u} \sigma_{k+u, j-u} \\
= & (-1)^{p} \sum_{j=1}^{n} \frac{\ln ^{n-j}(-2)}{(n-j) !} \sum_{u=0}^{j-1} C_{p+u-1}^{u}(-1)^{u} s_{p+u, j-u}+(-1)^{p} \\
& \times \sum_{j=0}^{n} \frac{\ln ^{n-j} 2 \ln ^{p+j}(-1)}{(n-j) !(p+j) !}, \\
S_{n, p}(2) & +\sum_{k=1}^{p} \frac{(-1)^{p-k} \ln ^{p-k}(-1)}{(p-k) !} \sum_{j=1}^{n} \frac{(-1)^{j} \ln ^{n-j} 2}{(n-j) !} \sigma_{k, j} \\
= & \sum_{j=1}^{n} \frac{\ln ^{n-j} 2}{(n-j) !} s_{j, p}+\frac{(-1)^{p} \ln ^{p}(-1) \ln ^{n} 2}{p ! n !} .
\end{aligned}
$$

By (49) we get

$$
\begin{aligned}
\sum_{j=1}^{n} \frac{\ln ^{n-j} 2}{(n-j) !}\left(\sum_{k=1}^{p} \frac{(-1)^{j}(-1)^{k} \ln ^{p-k}(-1)}{(p-k) !} \sigma_{k, j}\right. \\
\left.-\sum_{k=1}^{p} \frac{(-1)^{j} \ln ^{p-k}(-1)}{(p-k) !} \sum_{u=0}^{j-1} C_{k+u-1}^{u} \sigma_{k+u, j-u}\right) \\
=\sum_{j=1}^{n} \frac{\ln ^{n-j} 2}{(n-j) !}\left((-1)^{p} s_{j, p}-\frac{\ln ^{p+j}(-1)}{(p+j) !}\right. \\
\left.-\sum_{k=1}^{j} \frac{\ln ^{j-k}(-1)}{(j-k) !} \sum_{u=0}^{k-1} C_{p+u-1}^{u}(-1)^{u} s_{p+u, k-u}\right) .
\end{aligned}
$$

Noting the bracket part independent of $n$ in (50), it follows that

$$
\begin{aligned}
& \sum_{k=1}^{[(p+1) / 2]} \frac{\ln ^{p+1-2 k}(-1)}{(p+1-2 k) !} \sigma_{2 k-1, n} \\
& \quad+\sum_{k=1}^{p} \frac{\ln ^{p-k}(-1)}{(p-k) !} \sum_{j=1}^{n-1} C_{k+j-1}^{j} \sigma_{k+j, n-j} \\
& =\frac{(-1)^{n} \ln ^{p+n}(-1)}{(p+n) !}+(-1)^{n} \sum_{j=1}^{n} \frac{\ln ^{n-j}(-1)}{(n-j) !} \\
& \quad \times \sum_{u=0}^{j-1} C_{p+u-1}^{u}(-1)^{u} s_{p+u, j-u}-(-1)^{p+n} s_{n, p} .
\end{aligned}
$$


Taking into account the real and imaginary parts, we have

$$
\begin{aligned}
& \sum_{k=1}^{p} \frac{(-1)^{p-k} \pi^{2 p-2 k}}{(2 p+1-2 k) !}\left(2 \sigma_{2 k-1, n}+\sum_{j=1}^{n-1} C_{2 k+j-2}^{j} \sigma_{2 k-1+j, n-j}\right) \\
& =\delta_{n-1,2[(n-1) / 2]} \frac{(-1)^{p+(n+1) / 2} \pi^{2 p+n-1}}{(2 p+n) !} \\
& -(-1)^{n} \sum_{j=1}^{[n / 2]} \frac{(-1)^{j} \pi^{2 j-2}}{(2 j-1) !} \\
& \times \sum_{u=0}^{n-2 j} C_{2 p+u-1}^{u}(-1)^{u} s_{2 p+u, n+1-2 j-u} \\
& \sum_{k=1}^{p} \frac{(-1)^{p-k} \pi^{2 p-2 k}}{(2 p+1-2 k) !} \sum_{j=1}^{n-1} C_{2 k+j-1}^{j} \sigma_{2 k+j, n-j} \\
& =\delta_{n, 2[n / 2]} \frac{(-1)^{p+n / 2} \pi^{2 p+n}}{(2 p+n+1) !}-(-1)^{n} \sum_{j=1}^{[n / 2]} \frac{(-1)^{j} \pi^{2 j-2}}{(2 j-1) !} \\
& \times \sum_{u=0}^{n-2 j} C_{2 p+u}^{u}(-1)^{u} s_{2 p+u+1, n+1-2 j-u} \\
& \sum_{k=1}^{p} \frac{(-1)^{p-k} \pi^{2 p-2 k}}{(2 p-2 k) !} \sum_{j=1}^{n-1} C_{2 k+j-1}^{j} \sigma_{2 k+j, n-j} \\
& =\delta_{n, 2[n / 2]} \frac{(-1)^{p+n / 2} \pi^{2 p+n}}{(2 p+n) !}-(-1)^{n} s_{n, 2 p} \\
& +(-1)^{n} \sum_{j=0}^{[(n-1) / 2]} \frac{(-1)^{j} \pi^{2 j}}{(2 j) !} \\
& \times \sum_{u=0}^{n-2 j-1} C_{2 p+u-1}^{u}(-1)^{u} s_{2 p+u, n-2 j-u}, \\
& \sum_{k=1}^{p} \frac{(-1)^{p-k} \pi^{2 p-2 k}}{(2 p-2 k) !}\left(2 \sigma_{2 k-1, n}\right. \\
& \left.+\sum_{j=1}^{n-1} C_{2 k+j-2}^{j} \sigma_{2 k+j-1, n-j}\right) \\
& =(-1)^{n} s_{n, 2 p-1}+\delta_{n-1,2[(n-1) / 2]} \frac{(-1)^{p+(n+1) / 2} \pi^{2 p+n-1}}{(2 p+n-1) !} \\
& +(-1)^{n} \sum_{j=0}^{[(n-1) / 2]} \frac{(-1)^{j} \pi^{2 j}}{(2 j) !} \\
& \times \sum_{u=0}^{n-2 j-1} C_{2 p+u-2}^{u}(-1)^{u} s_{2 p+u-1, n-2 j-u} .
\end{aligned}
$$

In (52), letting $n=2$, we get that

$$
\begin{aligned}
\sigma_{2 p-1,2}= & \left(1-p-(2 p-1) 2^{-2 p-1}\right) \zeta(2 p+1) \\
& -\frac{1}{2} \sum_{k=1}^{p-1} \frac{(2 k-1)\left(1-2^{-2 k}\right)(-1)^{p-k} \pi^{2 p-2 k} \zeta(2 k+1)}{(2 p+1-2 k) !} \\
& +\sum_{k=1}^{p-1} \frac{(-1)^{p-k} \pi^{2 p-2 k}}{(2 p+1-2 k) !} \sigma_{2 k-1,2}, \\
\sigma_{2 p-1,2}= & \left(1-p+(2 p-1) 2^{-2 p-1}\right) \zeta(2 p+1) \\
& +\sum_{j=1}^{p-1} \zeta(1+j) \zeta(2 p-j) \\
& -\frac{1}{2} \sum_{k=1}^{p-1} \frac{(2 k-1)\left(1-2^{-2 k}\right)(-1)^{p-k} \pi^{2 p-2 k} \zeta(2 k+1)}{(2 p-2 k) !} \\
& -\sum_{k=1}^{p-1} \frac{(-1)^{p-k} \pi^{2 p-2 k}}{(2 p-2 k) !} \sigma_{2 k-1,2} \cdot
\end{aligned}
$$

Thus we have established two recursive formulas for $\sigma_{2 n-1,2}$. In (30), letting $z=1 / 2$, we have

$$
\begin{aligned}
a_{n, p} & +\sum_{j=1}^{n} \frac{(-1)^{n-j} \ln ^{n-j} 2}{(n-j) !} \sum_{v=0}^{p-1} C_{j+v-1}^{v} \sigma_{p-v, j+v} \\
= & \sum_{j=1}^{n} \frac{(-1)^{n-j} \ln ^{n-j} 2}{(n-j) !} s_{j, p}+\frac{(-1)^{n} \ln ^{n+p} 2}{(n+p) !} .
\end{aligned}
$$

In (33), letting $z=1 / 2$, we have

$$
\begin{aligned}
a_{n, p} & -\sum_{j=1}^{n} \frac{(-1)^{n-j} \ln ^{n-j} 2}{(n-j) !} \\
\times & \sum_{u=1}^{j} C_{p+j-1-u}^{j-u} \sigma_{u, p+j-u}=\frac{(-1)^{n} \ln ^{n+p} 2}{(n+p) !} .
\end{aligned}
$$

Equation (55) is just consistent with (9.11) in [6]. By (54) and (55), we have

$$
\begin{aligned}
\sum_{j=1}^{n} \frac{(-1)^{n-j} \ln ^{n-j} 2}{(n-j) !}\left(\sum_{u=0}^{j-1} C_{p+u-1}^{u} \sigma_{j-u, p+u}\right. \\
\\
\left.\quad+\sum_{v=0}^{p-1} C_{j+v-1}^{v} \sigma_{p-v, j+v}-s_{j, p}\right)=0 .
\end{aligned}
$$

Similarly, noting bracket part independent of $n$ in (56), we have

$$
\sum_{u=0}^{n-1} C_{p+u-1}^{u} \sigma_{n-u, p+u}+\sum_{v=0}^{p-1} C_{n+v-1}^{v} \sigma_{p-v, n+v}=s_{n, p},
$$

which is equivalent to (9.8) in [5]. 
TABLE 1: Time-consuming comparison of the algorithms (59), (4), (28), (30), (33), and (34) with PolyLog for different precistions $(n=4$, $p=5$, and $m=16$ ).

\begin{tabular}{lcccccc}
\hline & $z 0$ & $r$ & $T 1_{32}, T 2_{32}, T 1_{32} / T 2_{32}$ & err1, err2 & $T 1_{64}, T 2_{64}, T 1_{64} / T 2_{64}$ & errl, err2 \\
\hline$(59)$ & 0 & $1 / 8$ & $0.0654,0.0009,67.0$ & $0 . * 10^{-32}, 0 . * 10^{-31}$ & $0.1728,0.0019,88.5$ & $0 . * 10^{-64}, 0 . * 10^{-64}$ \\
$(59)$ & 0 & $1 / 4$ & $0.0673,0.0019,34.5$ & $0 . * 10^{-32}, 0 . * 10^{-32}$ & $0.1748,0.0039,44.7$ & $0 . * 10^{-64}, 0 . * 10^{-64}$ \\
$(4)$ & 1 & $1 / 8$ & $0.5136,0.0107,47.8$ & $0 . * 10^{-26}, 0 . * 10^{-30}$ & $1.7880,0.0195,91.5$ & $0 . * 10^{-58}, 0 . * 10^{-62}$ \\
$(4)$ & 1 & $1 / 4$ & $0.4628,0.0156,29.6$ & $0 . * 10^{-25}, 0 . * 10^{-29}$ & $1.5361,0.0292,52.4$ & $0 . * 10^{-57}, 0 . * 10^{-61}$ \\
$(28)$ & 10 & 1 & $0.4326,0.0185,23.3$ & $0 . * 10^{-30}, 0 . * 10^{-29}$ & $1.2070,0.0380,31.6$ & $0 . * 10^{-62}, 0 . * 10^{-61}$ \\
$(28)$ & 100 & 10 & $0.4472,0.0195,22.8$ & $0 . * 10^{-31}, 0 . * 10^{-29}$ & $1.1201,0.0380,29.4$ & $0 . * 10^{-63}, 0 . * 10^{-61}$ \\
$(30)$ & 1 & $1 / 8$ & $0.5117,0.0195,26.1$ & $0 . * 10^{-26}, 0 . * 10^{-30}$ & $1.7988,0.0380,47.2$ & $0 . * 10^{-58}, 0 . * 10^{-62}$ \\
$(30)$ & 1 & $1 / 4$ & $0.4658,0.0292,15.8$ & $0 . * 10^{-25}, 0 . * 10^{-29}$ & $1.5361,0.0595,25.7$ & $0 . * 10^{-57}, 0 . * 10^{-61}$ \\
$(33)$ & -1 & $1 / 8$ & $0.4091,0.0449,9.1$ & $0 . * 10^{-25}, 0 . * 10^{-29}$ & $1.7275,0.0957,18.0$ & $6 . * 10^{-57}, 0 . * 10^{-61}$ \\
$(33)$ & -1 & $1 / 4$ & $0.4111,0.0468,8.7$ & $0 . * 10^{-25}, 0 . * 10^{-29}$ & $1.6464,0.1826,9.0$ & $6 . * 10^{-57}, 0 . * 10^{-61}$ \\
$(34)$ & -10 & 1 & $0.4423,0.0156,28.3$ & $0 . * 10^{-29}, 0 . * 10^{-29}$ & $1.3437,0.0302,44.3$ & $0 . * 10^{-61}, 0 . * 10^{-61}$ \\
$(34)$ & -100 & 10 & $0.4443,0.0156,28.4$ & $0 . * 10^{-30}, 0 . * 10^{-30}$ & $1.0556,0.0302,34.8$ & $0 . * 10^{-63}, 0 . * 10^{-62}$ \\
\hline
\end{tabular}

TABLE 2: Time-consuming comparison of the algorithms (59), (4), (28), (30), (33), and (34) with PolyLog for different precistions $(n=4$, $p=5$, and $m=16$ ).

\begin{tabular}{lcccccc}
\hline & $z 0$ & $r$ & $T 1_{96}, T 2_{96}, T 1_{96} / T 2_{96}$ & err1, err2 & $T 1_{128}, T 2_{128}, T 1_{128} / T 2_{128}$ & errl, err2 \\
\hline$(59)$ & 0 & $1 / 8$ & $0.2236,0.0039,57.2$ & $0 . * 10^{-96}, 0 . * 10^{-96}$ & $0.5664,0.0048,116.0$ & $0 . * 10^{-128}, 0 . * 10^{-128}$ \\
$(59)$ & 0 & $1 / 4$ & $0.2275,0.0058,38.8$ & $0 . * 10^{-96}, 0 . * 10^{-96}$ & $0.5664,0.0078,72.5$ & $0 . * 10^{-128}, 0 . * 10^{-128}$ \\
$(4)$ & 1 & $1 / 8$ & $5.8251,0.0302,192.4$ & $0 . * 10^{-89}, 0 . * 10^{-94}$ & $8.7773,0.0410,214$. & $0 . * 10^{-118}, 0 . * 10^{-126}$ \\
$(4)$ & 1 & $1 / 4$ & $3.2138,0.0458,70.0$ & $0 . * 10^{-89}, 0 * 10^{-93}$ & $5.9726,0.0634,94.0$ & $0 . * 10^{-121}, 0 . * 10^{-125}$ \\
$(28)$ & 10 & 1 & $2.2666,0.0585,38.6$ & $0 . * 10^{-94}, 0 * 10^{-93}$ & $6.1044,0.0791,77.1$ & $0 . * 10^{-126}, 0 . * 10^{-125}$ \\
$(28)$ & 10 & 10 & $2.2167,0.0576,38.4$ & $0 . * 10^{-95}, 0 . * 10^{-93}$ & $4.1611,0.0781,53.2$ & $0 . * 10^{-127}, 0 . * 10^{-125}$ \\
$(30)$ & 1 & $1 / 8$ & $3.7890,0.0595,63.6$ & $0 . * 10^{-89}, 0 . * 10^{-94}$ & $6.6630,0.0820,81.2$ & $0 . * 10^{-118}, 0 . * 10^{-126}$ \\
$(30)$ & 1 & $1 / 4$ & $3.1523,0.0917,34.3$ & $0 . * 10^{-89}, 0 . * 10^{-93}$ & $26.0390,6.3847,4.0$ & $0 . * 10^{-121}, 0 . * 10^{-125}$ \\
$(33)$ & -1 & $1 / 8$ & $3.3681,5.0224,0.67$ & $0 . * 10^{-89}, 0 . * 10^{-93}$ & $5.9814,5.0312,1.18$ & $0 . * 10^{-121}, 0 . * 10^{-125}$ \\
$(33)$ & -1 & $1 / 4$ & $1.4921,4.8085,0.31$ & $0 . * 10^{-89}, 0 . * 10^{-93}$ & $5.5576,4.8798,1.1$ & $0 . * 10^{-121}, 0 . * 10^{-125}$ \\
$(34)$ & -10 & 1 & $2.3085,0.0478,48.2$ & $0 . * 10^{-93}, 0 . * 10^{-93}$ & $5.6611,0.0664,85.2$ & $0 . * 10^{-125}, 0 . * 10^{-125}$ \\
$(34)$ & -100 & 10 & $2.2587,0.0488,46.2$ & $0 . * 10^{-95}, 0 . * 10^{-94}$ & $4.0634,0.0664,61.1$ & $0 . * 10^{-127}, 0 . * 10^{-126}$ \\
\hline
\end{tabular}

\section{High Accuracy and Fast Calculation for the Nielsen Generalized Polylogarithm $S_{n, p}(z)$}

In this section, we apply the transformation formulas established previously to design algorithms of high accuracy and fast calculation for the Nielsen generalized polylogarithm $S_{n, p}(z)$. In Mathematica, there is an internal command, PolyLog $[n, p, z]$, designed to calculate the Nielsen generalized polylogarithm $S_{n, p}(z)$. When $|z|<1$, and the integers $n, p \geq 1$ in the Nielsen generalized polylogarithm $S_{n, p}(z)$, using (1) and

$$
\ln ^{p}(1-t)=(-1)^{p} p ! \sum_{m=p}^{\infty} \frac{t^{m} H_{m-1}^{(p-1)}}{m}
$$

where

$$
\begin{gathered}
H_{m}^{(k+1)}=\sum_{j=k+1}^{m} \frac{1}{j} H_{j-1}^{(k)}, \\
H_{m}^{(1)}=H_{m}, \\
H_{m}^{(0)}=1,
\end{gathered}
$$

satisfying the following recursive formula,

$$
H_{n}^{(k)}=H_{n-1}^{(k)}+\frac{1}{n} H_{n-1}^{(k-1)},
$$

we have

$$
S_{n, p}(z)=\sum_{m=p}^{\infty} \frac{z^{m} H_{m-1}^{(p-1)}}{m^{n+1}} .
$$

Concerning the computational speed and accuracy, we make a comparison between PolyLog and (59), (4), (28), (30), (33), and (34). We will make the comparison in $m$ welldistributed points from a circle with the center $z_{0}$ and the radius $r$. Numerical results are given in Tables 1 and 2 .

Here $\mathrm{T} 1_{P}$, err 1 are the average running time and the relative error of PolyLog $[n, p, N[z, \operatorname{Prec}]]$, while $T 2_{P}$, err 2 are the average running time and the relative error of (59), (4), (28), (30), (33), and (34) in precision $P$, respectively. We always use second as the unit of time.

Seen from Table 1, when the precision $P \leqslant 64$, (59), (4), (28), (30), (33), and (34) are faster than the PolyLog. Seen from Table 2, in addition to (33), with improved precision, it 
is more obvious that the calculation speed of (59), (4), (28), (30), and (34) is faster than PolyLog.

Remark 8. When $z_{1}=1 / 2+\sqrt{3} i / 2$ or $z_{2}=1 / 2-\sqrt{3} i / 2$, since $\left|1-z_{i}\right|=\left|1 /\left(1-z_{i}\right)\right|=\left|z_{i} /\left(z_{i}-1\right)\right|=1, i=1,2$, is calculated as a value, (59), (4), (28), (30), (33), and (34) cannot be used. But so far we did not find such a transformation $\left|\left(a+z_{i}\right) /\left(b+z_{i}\right)\right|<$ $1, i=1,2$. Although in the literature [6] the author gives some transformation $z \rightarrow(a+z) /(b+z)$, it essentially still belongs to the above type of transformation. For example, in [6], the author gives the following results:

$$
\begin{aligned}
& L_{n, p}\left(\frac{a+z}{b+z}\right) \\
& =s_{n, p}-\sum_{k=0}^{p} C_{n+p-k-1}^{n-1} M_{k+1, n+p-k-1}\left(\frac{b-a}{b+z}\right) \\
& \quad+L_{p+1, n-1}\left(\frac{b-a}{b+z}\right),
\end{aligned}
$$

where $M_{n, p}(z)=\left((-1)^{n+p-1} /(n-1) ! p !\right) \int_{0}^{z}\left(\ln ^{n-1} t \ln ^{p}(1+\right.$ $t) / t) d t$. If the $(b-a) /(b+z)$ into $z$, (62) becomes

$$
\begin{aligned}
& L_{n, p}(1-z) \\
& \quad=s_{n, p}-\sum_{k=0}^{p} C_{n+p-k-1}^{n-1} M_{k+1, n+p-k-1}(z)+L_{p+1, n-1}(z),
\end{aligned}
$$

this is clearly the type of (4).

\section{Conclusions}

In this paper, for the Nielsen generalized polylogarithm $S_{n, p}(z)$, the formulas for the transformations $z \rightarrow$ $p(z), p(z)=1-z, 1 / z, 1 /(1-z), z /(z-1)$, and $(1-z) / z$ have been established, and the last three transformation formulas are new. It is worthy to note that the transformation formula (28) is faster than (1.5) in [5] after taking into account numerical calculation. By use of these transformation formulas established above, new fast algorithms for Nielsen generalized polylogarithm $S_{n, p}(z)$ can be designed. Numerical results show that when $\left.\mid z-z_{i}\right]>2 / 5$, where $z_{1}=1 / 2+i \sqrt{3} / 2, z_{2}=1 / 2-i \sqrt{3} / 2$, the new algorithm is superior to Mathematica's internal functions PolyLog tens of times faster or even a hundred times. For $s_{n, p}=S_{n, p}(1)$, we have also given a new recursive formula (36). For $a_{n, p}=S_{n, p}(1 / 2)$ and $\sigma_{n, p}=S_{n, p}(-1)$, we have got some new identities, some of which are different from those in [5].

\section{Conflict of Interests}

The authors declare that there is no conflict of interests regarding the publication of this paper.

\section{Acknowledgment}

This work is supported by National Natural Science Foundation of China (61379009).

\section{References}

[1] I. S. Gradshteyn and I. M. Ryzhik, Table of Integrals, Series and Products, Academic Press, New York, NY, USA, 1980.

[2] H. Cheng and T. Wu, Expanding Protons: Scattering at High Energies, The MIT Press, Cambridge, Mass, USA, 1987.

[3] W. Maier and H. Kiesewetter, Funktionalgleichungen Mit Analytischen Lösungen, Deutscher Verlag der Wissenschaften, Berlin, Germany, 1971.

[4] K. S. Kölbig, J. A. Mignaco, and E. Remiddi, "On Nielsen's generalized polylogarithms and their numerical calculation," BIT, vol. 10, no. 1, pp. 38-73, 1970.

[5] K. S. Kölbig, "Nielsen's generalized polylogarithms," The SIAM Journal on Mathematical Analysis, vol. 17, pp. 1232-1258, 1986.

[6] H. Jacques, Lectures on Cauchy's Problem in Linear Partial Differential Equations, Dover Phoenix, New York, NY, USA, 1923.

[7] D. F. Paget, "The numerical evaluation of Hadamard finite-part integrals," Numerische Mathematik, vol. 36, no. 4, pp. 447-453, 1981.

[8] B. Bialecki, "A sinc quadrature rule for Hadamard finite-part integrals," Numerische Mathematik, vol. 57, no. 1, pp. 263-269, 1990. 


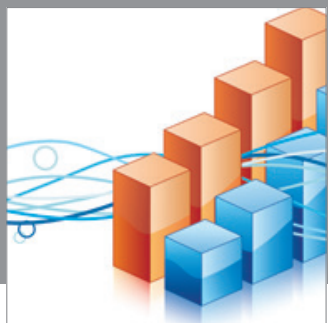

Advances in

Operations Research

mansans

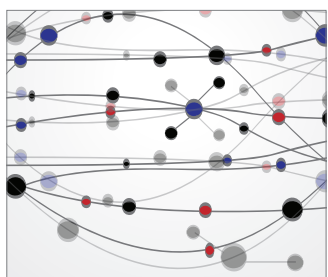

The Scientific World Journal
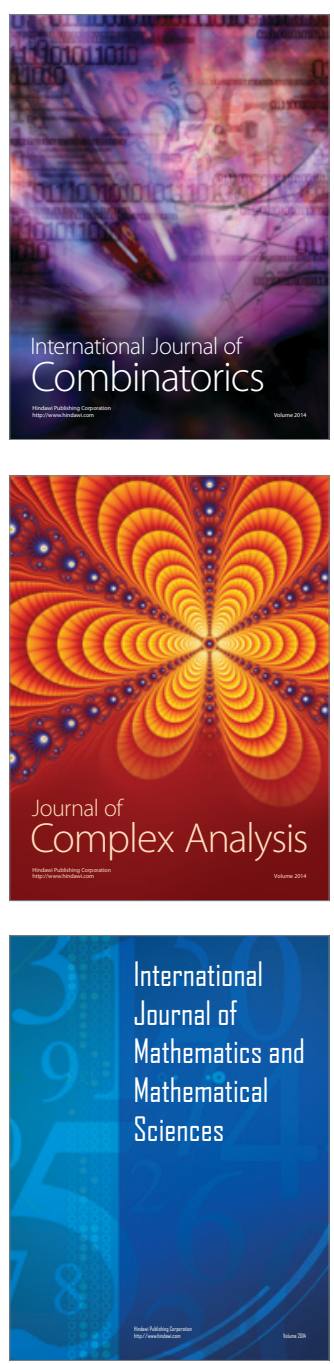
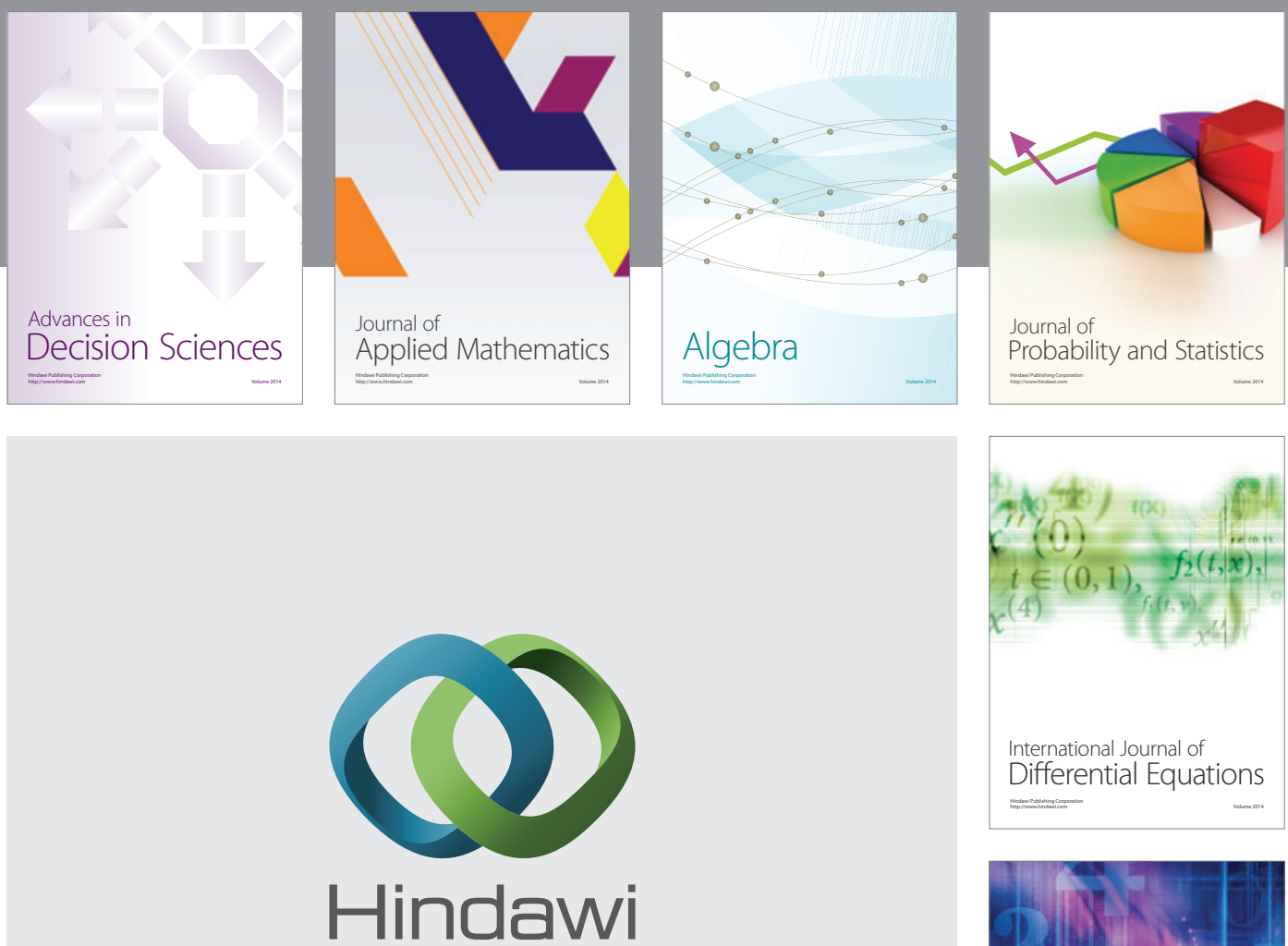

Submit your manuscripts at http://www.hindawi.com
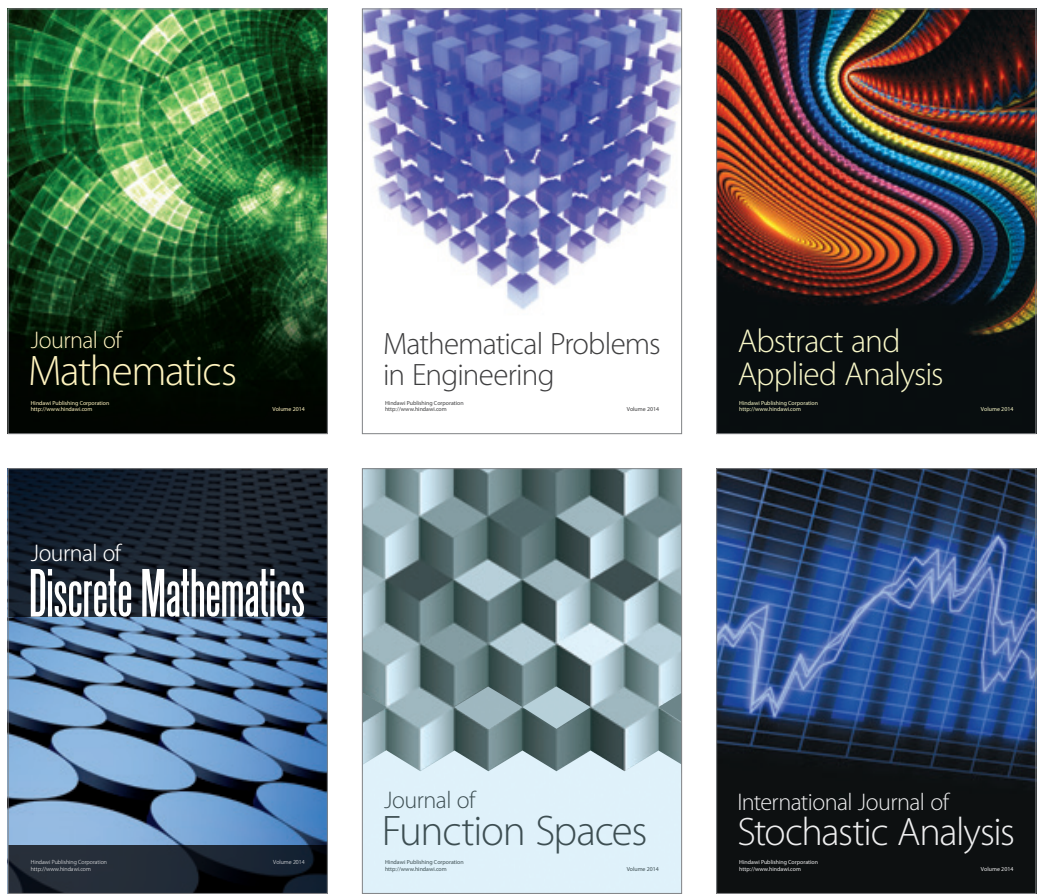

Journal of

Function Spaces

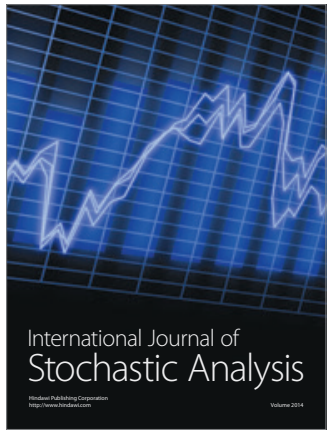

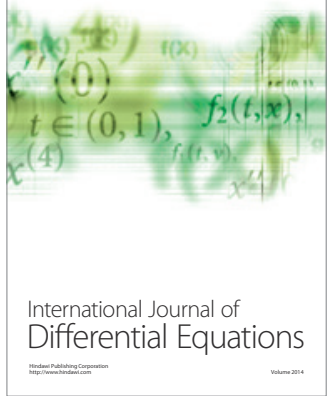
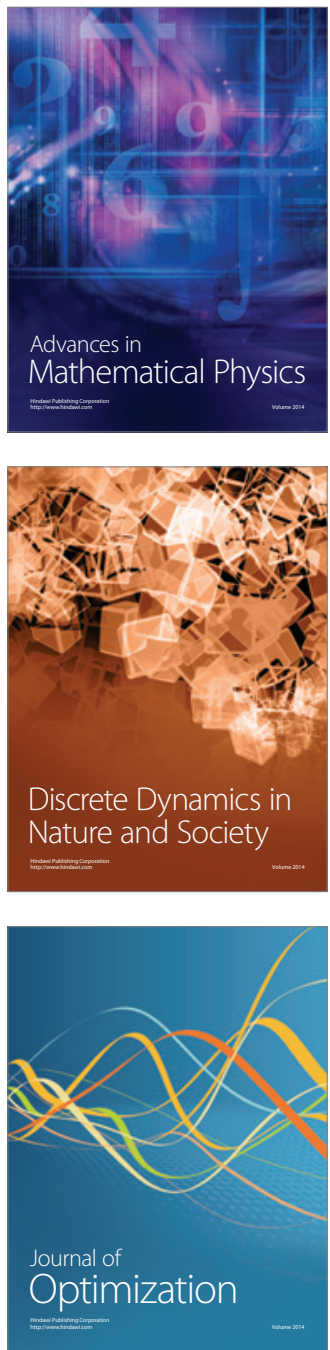